\title{
CalCon 2013
}

August, 2013

\section{Global Precipitation Measurement Microwave Imager (GMI) Pre-flight Noise Diode Performance David Draper}




\section{Global Precipitation Measurement (GPM) Mission}
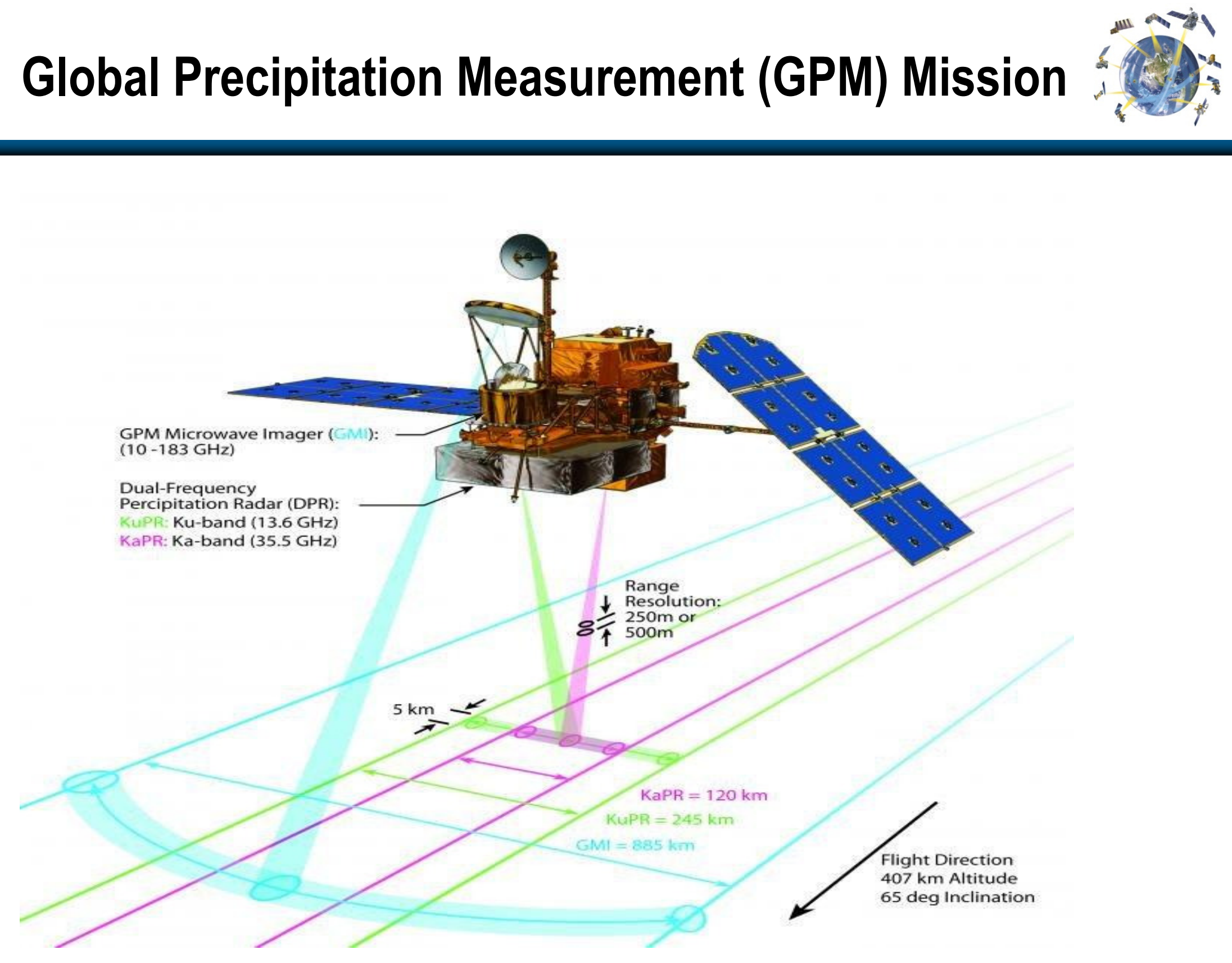


\section{GMI Is a State-of-the-Art Microwave Radiometer}

- Radiometric

-13 channels at 7 frequencies : $10.65,18.7,23.8$, $36.5,89,166$ and 183

- Spatial

- Main reflector is 1.22 meters compared to TRMM and SSMIS 0.8 meter reflector providing improved spatial resolution

- Calibration

- GMI will be the calibration standard for all orbiting radiometers used for GPM rain retrieval

- Many design features were incorporated to improve calibration including isolation of the hot load, the size of the cold sky reflector and the use of noise diodes as an additional calibration source

- Mechanical

- Compact self contained design allows for easy accommodation on the GPM spacecraft and future spacecraft

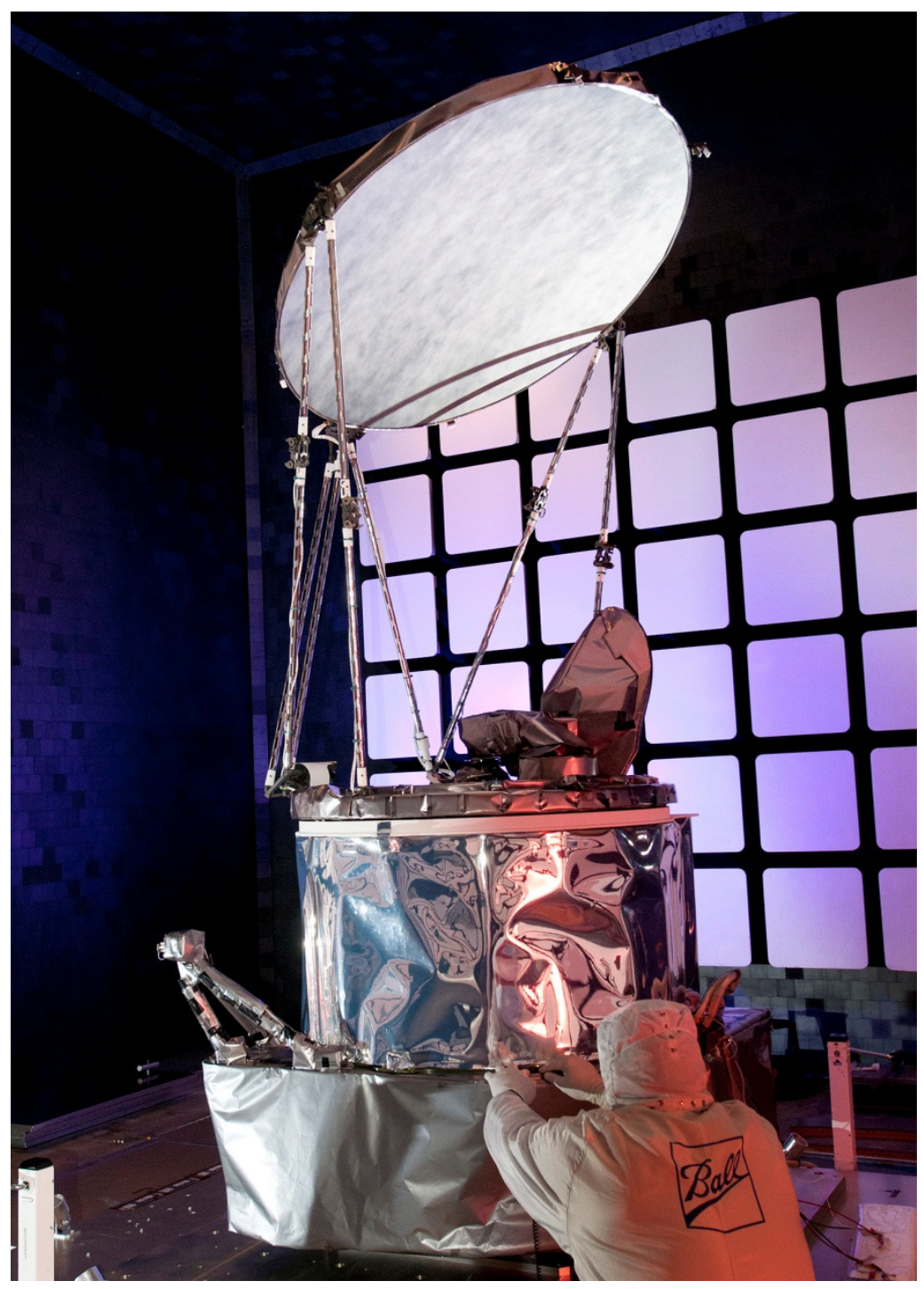




\section{Calibration Source Comparison}

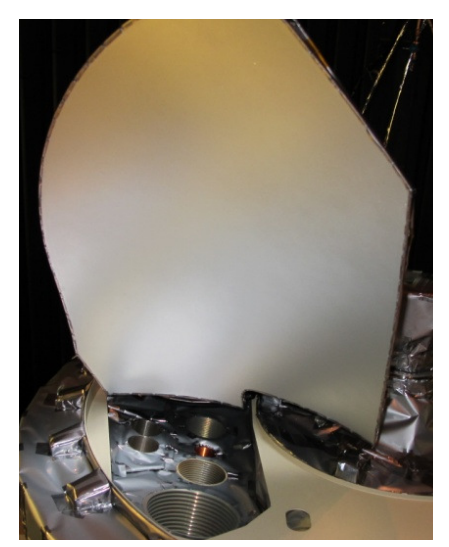

Flight Cold Sky Reflector

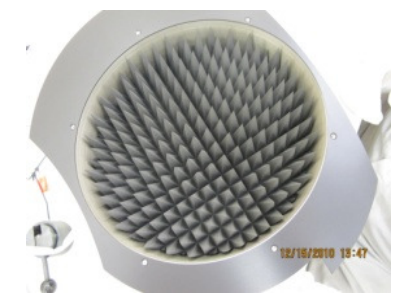

Flight Hot Load
- Cold sky reflector (space)

- Well defined vicarious calibration source

- Potential errors

* Spacecraft occultation

* Reflector spill over

* RFI

- Warm load (black body)

- Physical standard

- Potential errors

* Thermal gradients (heaters or solar intrusion)

* Incomplete beam filling

- Imperfect emissivity
- Noise sources

- Electronic generator

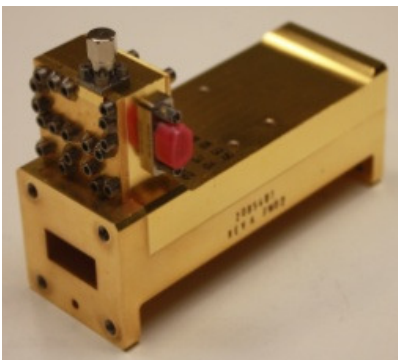

Noise Source

- Potential errors

* Pre-launch calibration

* Temperature error

* Non-thermal drift/jump

- Injected inline

- Measures gain

- Low thermal coefficient 


\section{Noise Sources Located Inline with Feed Horns}

- Calibration sources inline w/ feed waveguides

- Calibrate gain/nonlinearity ever other scan

- 36-GHz

- 18/23-GHz

- 10-GHz
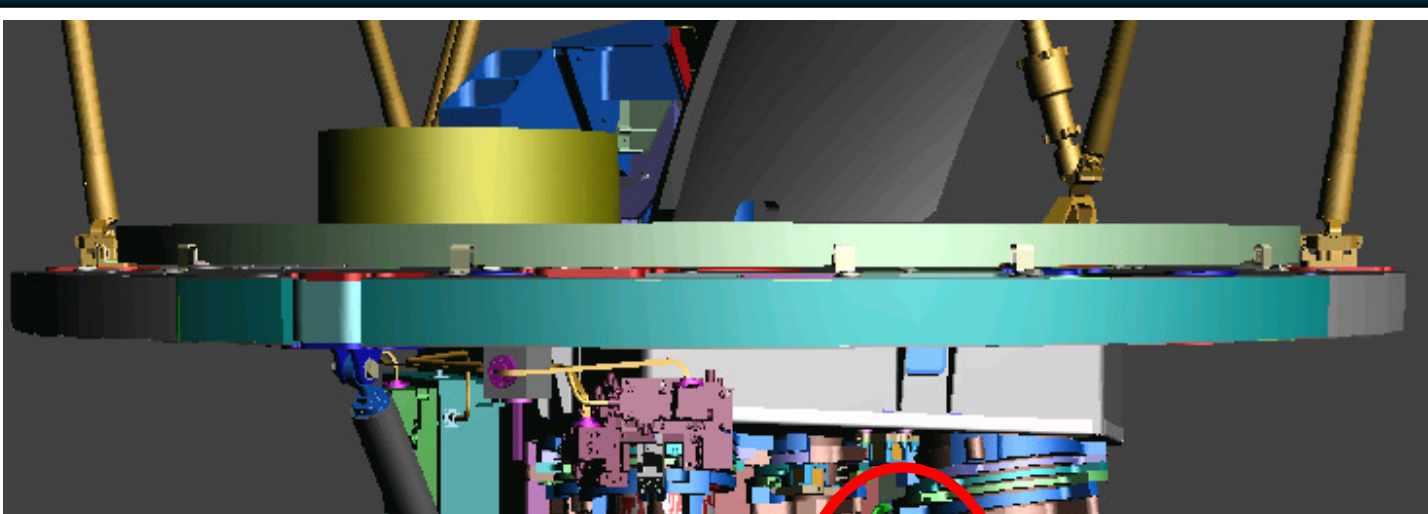
Noise Sources cover 10.7 to $36.5 \mathrm{GHz}$
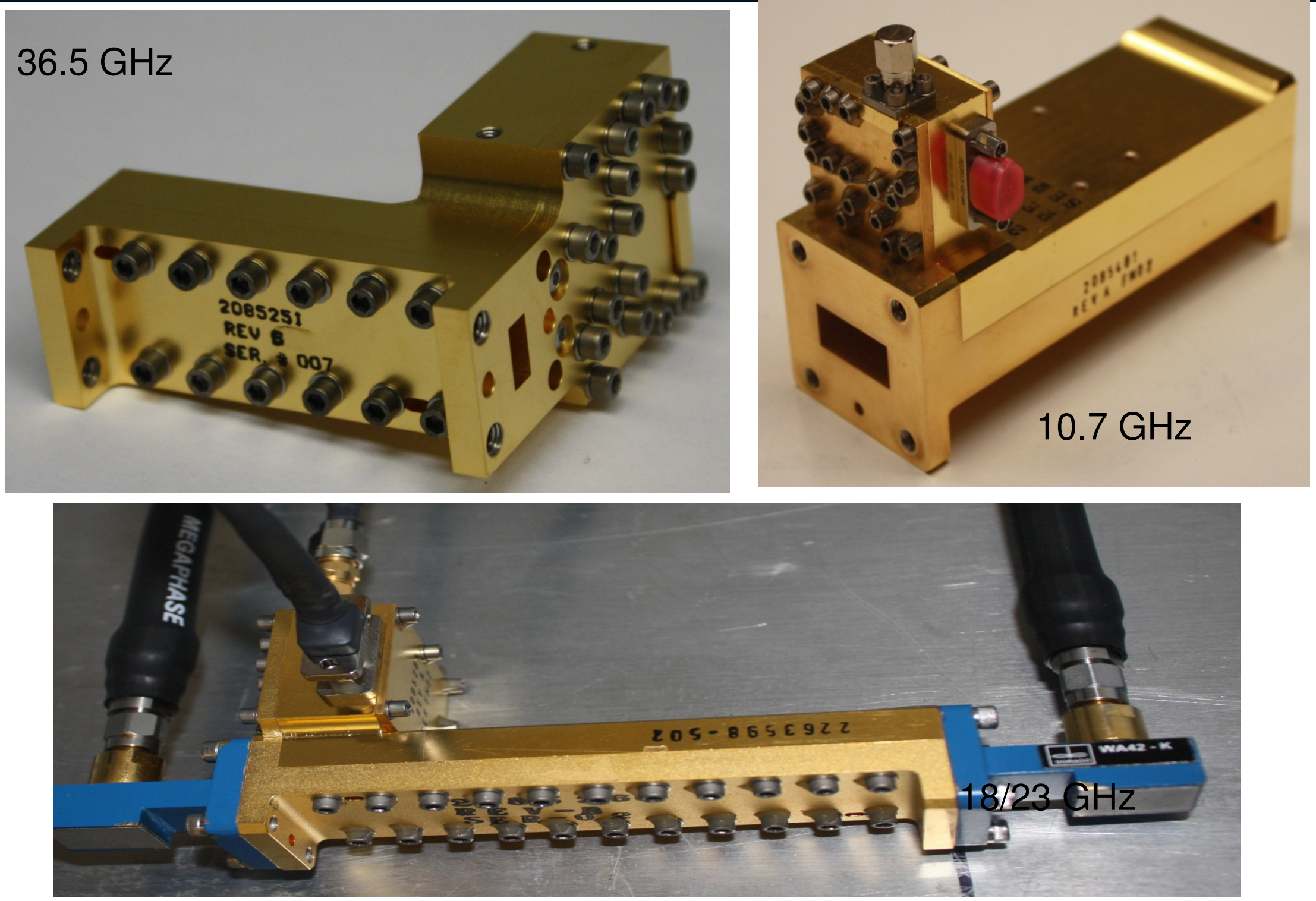


\section{Non-linearity and Noise Diode Excess Temperature can be derived from 4-point calibration}

- Four calibration points are measured: 1) Cold, 2) Cold + Noise, 3) Hot and 4) Hot + Noise

- Four calibration points provide four pieces of information

- Offset

- Gain (g)

- Non-linearity (Tnl)

- Noise Diode Excess Temperature (Tnd)

- The Noise Diode Excess Temperature can be trended

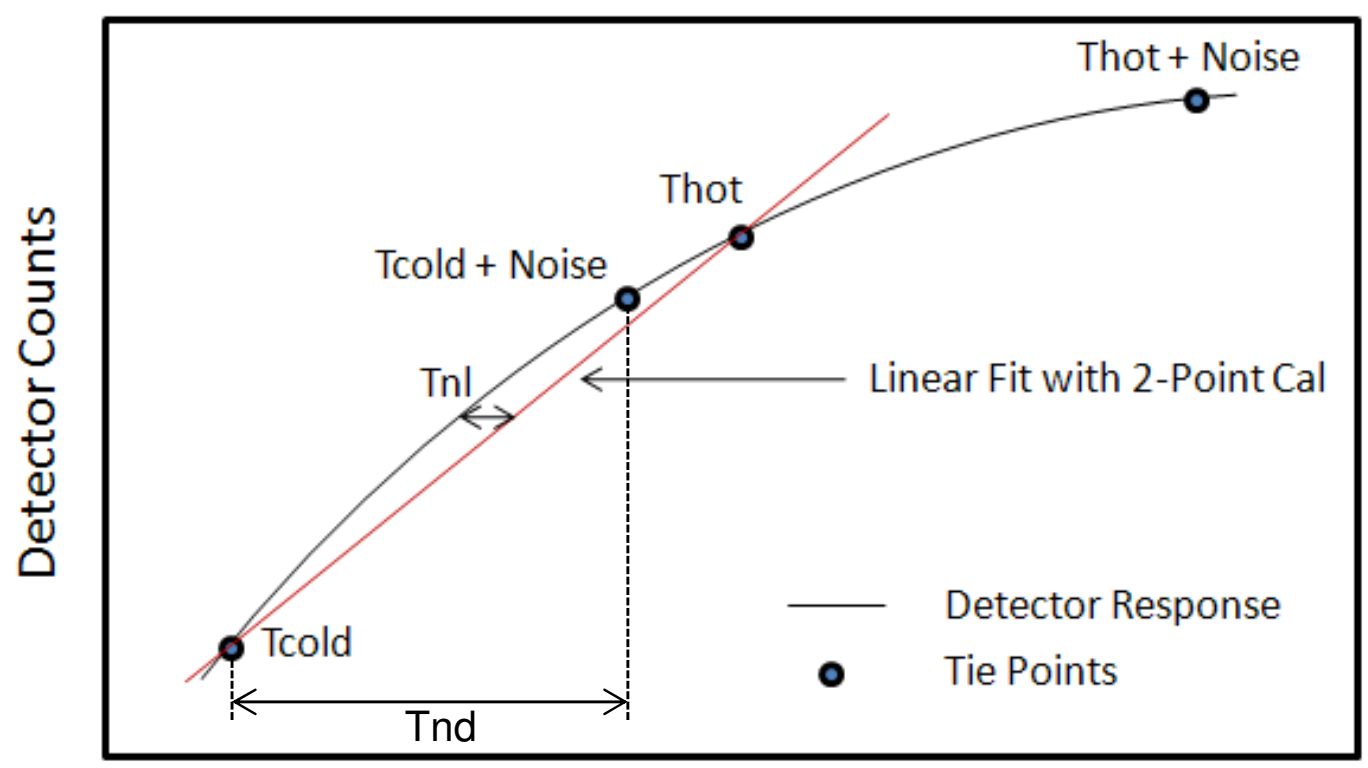

Input Scene Temperature 


\section{Using the noise diodes as a backup-calibration system}

- The primary hot/cold system + noise diodes provide 3 ways to calibrate

- Primary calibration:

- Hot and Cold Views

- Backup calibration:

- Cold and Cold + Noise (replaces hot load)

- Hot and Hot + Noise (replaces cold sky)

Primary Calibration
$T_{a}=T_{c}+\frac{C_{s}-C_{c}}{g}+u \frac{\left(C_{s}-C_{c}\right)\left(C_{s}-C_{h}\right)}{g^{2}}$
where $g=\left(C_{h}-C_{c}\right) /\left(T_{h}-T_{c}\right)$

\section{Backup Calibration with Cold and Cold+Noise}

$$
\begin{aligned}
& T_{a 1}=T_{c}+\frac{C_{s}-C_{c}}{g_{1}}+u \frac{\left(C_{s}-C_{c}\right)\left(C_{s}-C_{c n}\right)}{g_{1}^{2}} \\
& \text { where } g_{1}=\left(C_{c n}-C_{c}\right) / T_{n d-\text { trended }}
\end{aligned}
$$

\section{Backup Calibration with Hot and Hot+Noise}

$$
\begin{aligned}
& T_{a 2}=T_{h}+\frac{C_{s}-C_{h}}{g_{2}}+u \frac{\left(C_{s}-C_{h}\right)\left(C_{s}-C_{h n}\right)}{g_{2}^{2}} \\
& \text { where } g_{2}=\left(C_{h n}-C_{h}\right) / T_{n d-\text { trended }}
\end{aligned}
$$




\section{GMI Ground Calibration Test}

- Radiometer through-the-feed testing performed at the instrument level

- Main reflector is removed

- Test performed in the thermal vacuum chamber

- Instrument is rotating

- Instrument views three calibration loads, once per scan

- Flight Hot Load

- GSE Cold Calibration Target

- GSE Variable Temperature ("Scene") Target
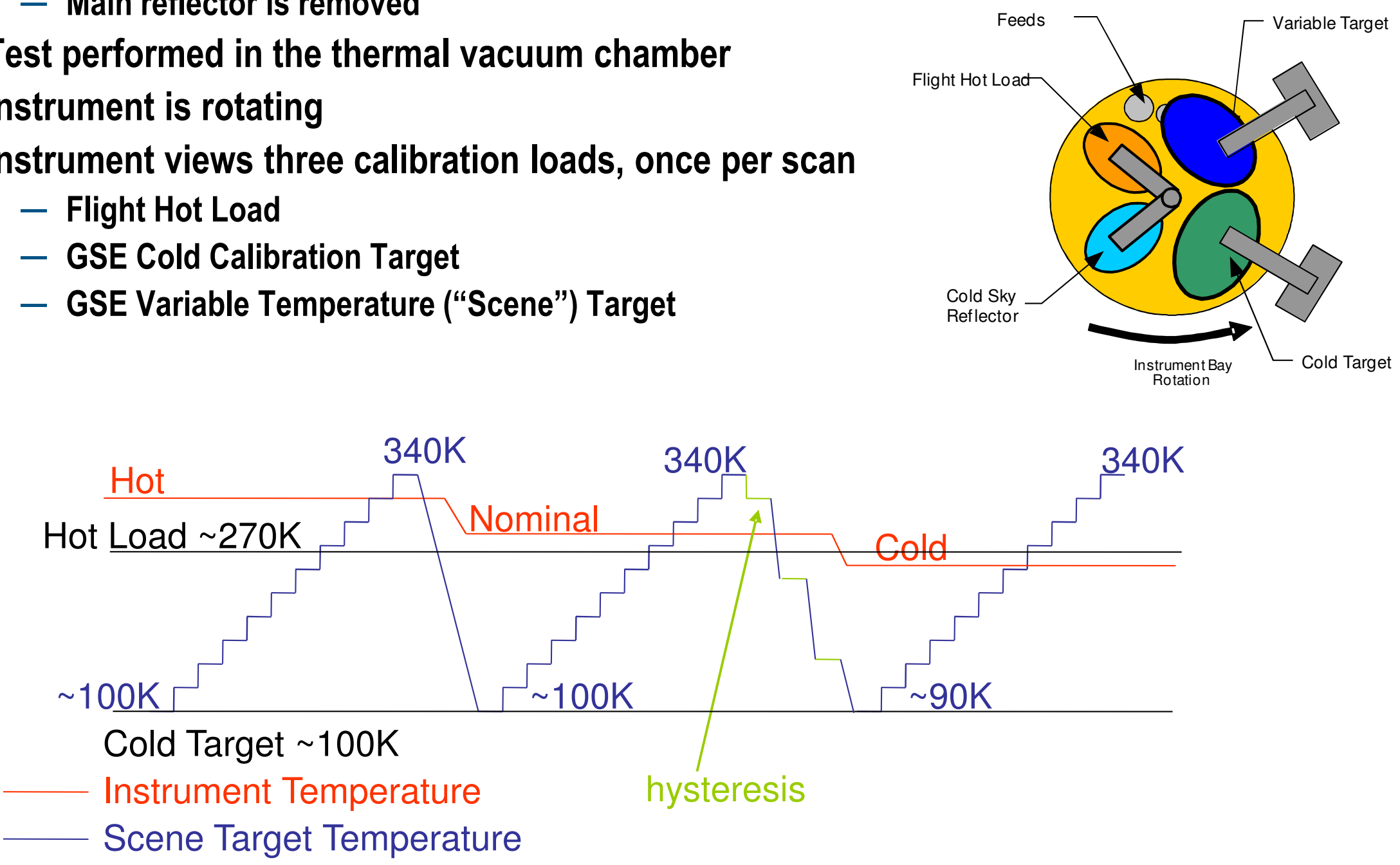


\section{"Backup Calibration" finds cold target radiometric errors during GMI calibration test}

- During GMI calibration test, the cold target exhibited "larger than expected" gradients

- Gradients were modeled and removed analytically, but there were still residual gradient errors

- Using the hot and hot + noise backup calibration, the cold target temperature can be measured

- The residual error was removed by scaling the gradient correction for the cold target
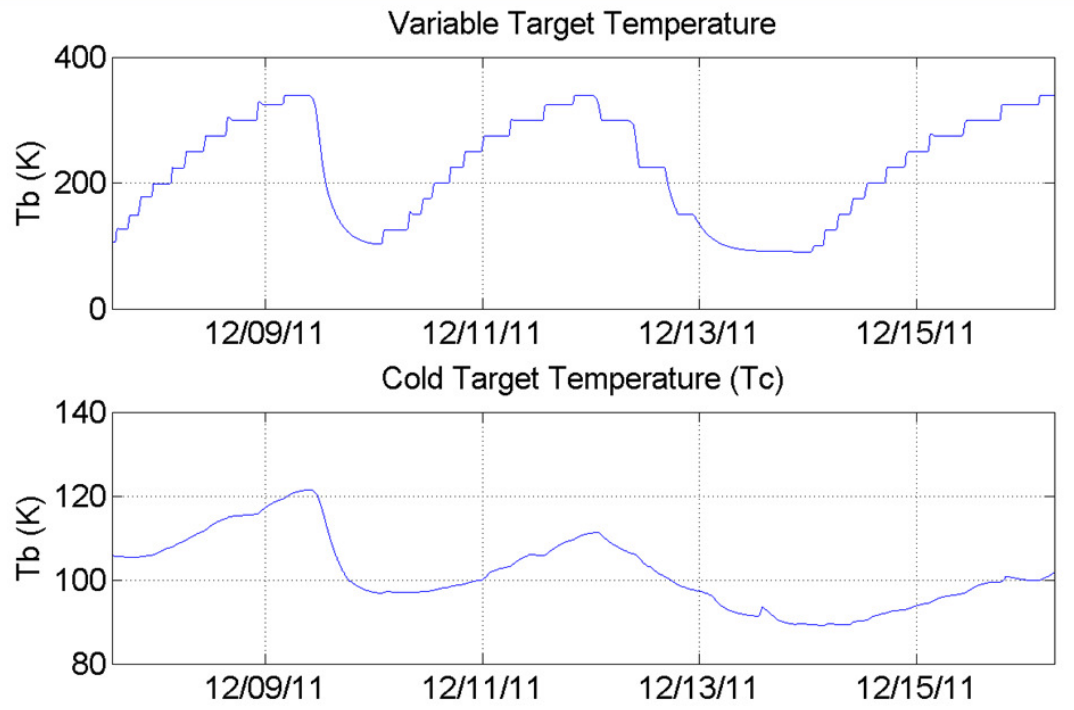

Cold Target Temp from Hot Load \& Noise Diodes (Tc2)

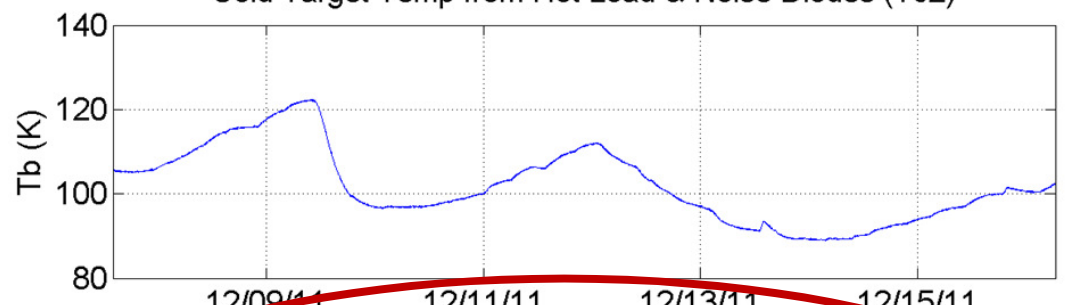

Cold Target Residual Error

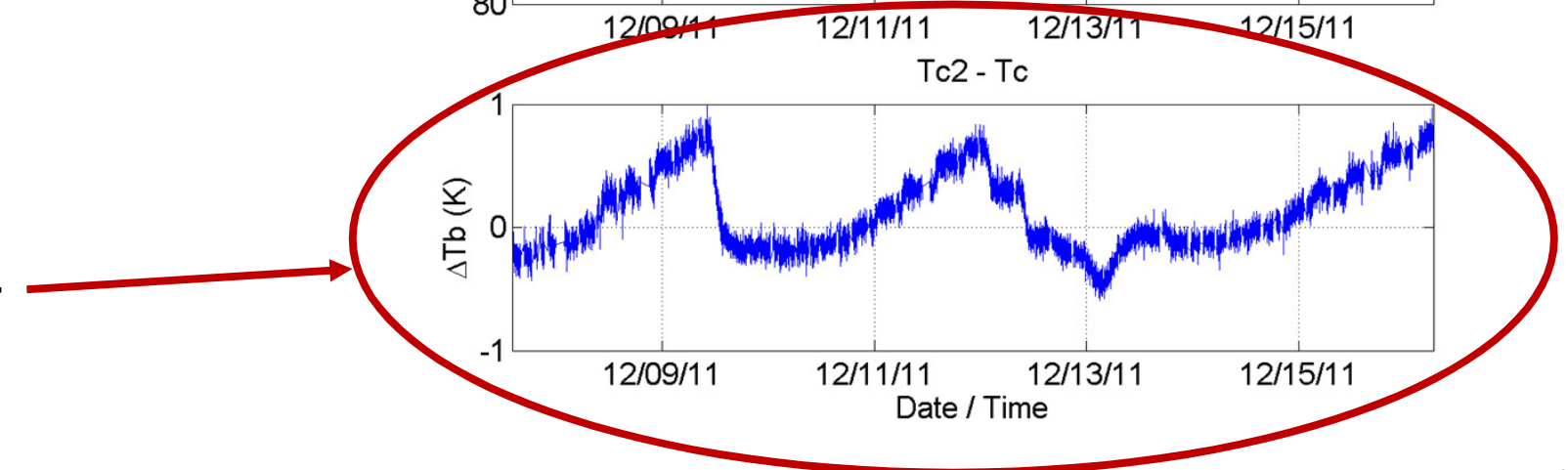




\section{Noise Diode Stability critical for "backup calibration"}

- The stability of the noise diode influences how predictably the excess temperature can be known

- From the calibration test, the relationship between the noise diode physical temperature and excess temperature can be determined.

- The deviation from a second order fit over the test gives an estimate of the "predictability" of the noise diode excess temperature.

Estimated stability of the GMI noise diodes.

\begin{tabular}{|c|c|c|}
\hline \multicolumn{3}{|c}{ All numbers are 3-sigma } \\
\hline Channel & $\begin{array}{c}\text { Tnd Stability } \\
\text { (Estimated w/o Cold } \\
\text { Correction) }\end{array}$ & $\begin{array}{c}\text { Tnd Stability } \\
\text { (Estimated w/ Cold } \\
\text { Correction) }\end{array}$ \\
\hline $10.65 \mathrm{GHz}$ V & $2.6 \mathrm{~K}$ & $2.6 \mathrm{~K}$ \\
\hline $10.65 \mathrm{GHz} \mathrm{H}$ & $1.2 \mathrm{~K}$ & $1.0 \mathrm{~K}$ \\
\hline $18.7 \mathrm{GHz} \mathrm{V}$ & $1.7 \mathrm{~K}$ & $0.4 \mathrm{~K}$ \\
\hline $18.7 \mathrm{GHz} \mathrm{H}$ & $1.7 \mathrm{~K}$ & $0.4 \mathrm{~K}$ \\
\hline $23.8 \mathrm{GHz} \mathrm{V}$ & $1.4 \mathrm{~K}$ & $0.4 \mathrm{~K}$ \\
\hline $36.64 \mathrm{GHz} \mathrm{V}$ & $1.6 \mathrm{~K}$ & $0.8 \mathrm{~K}$ \\
\hline $36.64 \mathrm{GHz} \mathrm{H}$ & $1.4 \mathrm{~K}$ & $0.7 \mathrm{~K}$ \\
\hline
\end{tabular}
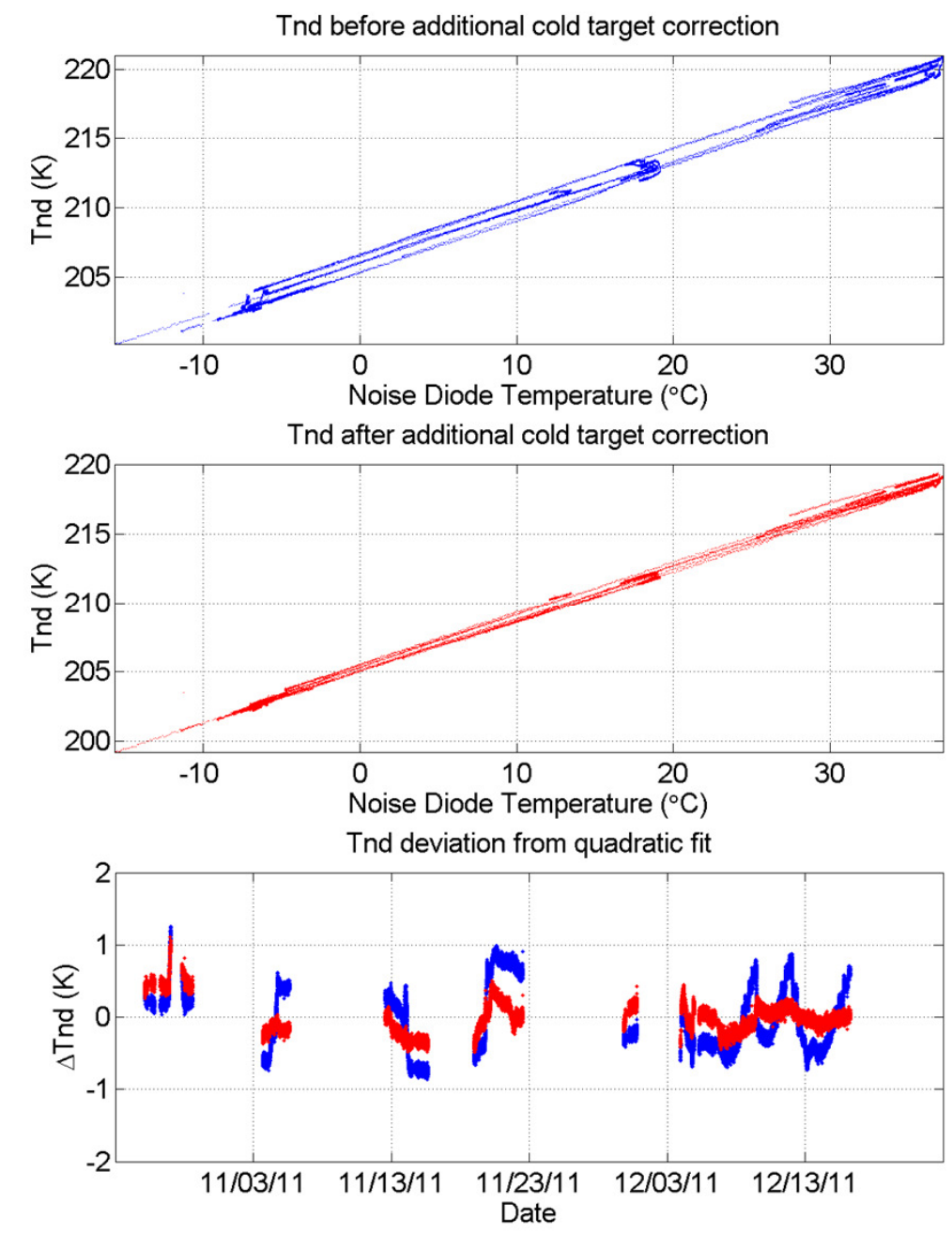


\section{Conclusions}

- GMI Noise Diodes will be used for the following:

- Trending non-linearity

- Identifying transient calibration errors

- As a backup to calibrate the instrument in case of calibration anomalies

- The GMI calibration test showed that the noise diodes will be "trendable" to within about $1 \mathrm{~K}$ for all but the $10 \mathrm{GHz} \mathrm{V}$-pol channel

- The $10 \mathrm{GHz}$ V-pol channel noise diode will likely stabilize on orbit, providing similar performance as the other noise diodes. 